\title{
Hypocoiled Umbilical Cord
}

National Cancer Institute

\section{Source}

National Cancer Institute. Hypocoiled Umbilical Cord. NCI Thesaurus. Code C117355.

Umbilical cord with apparent reduced helical coiling of the arteries around the vein. 